Cahiers de recherches médiévales

Journal of medieval studies

15 | 2008

La Tentation du parodique dans la littérature médiévale

\title{
Parodies en scène
}

Textes et contextes dans le théâtre de Pierre de Lesnauderie (Caen, 1493-1496)

\section{Estelle Doudet}

\section{(2) OpenEdition \\ Journals}

Édition électronique

URL : https://journals.openedition.org/crm/5563

DOI : $10.4000 / \mathrm{crm} .5563$

ISSN : 1955-2424

Éditeur

Honoré Champion

Édition imprimée

Date de publication : 20 juin 2008

Pagination : $31-44$

ISSN : 1272-9752

Référence électronique

Estelle Doudet, «Parodies en scène », Cahiers de recherches médiévales [En ligne], 15 | 2008, mis en ligne le 20 juin 2011, consulté le 15 décembre 2022. URL : http://journals.openedition.org/crm/5563 ; DOI : https://doi.org/10.4000/crm.5563 


\title{
酶M
}

\author{
Parodies en scène. \\ Textes et contextes dans le théâtre de Pierre de Lesnauderie \\ (Caen, 1493-1496)
}

\begin{abstract}
Parody is usually described as a textual phenomena, a comic travesty of written and well-known authorities. The strong mental frames of Western Medieval Culture designate this period as especially favourable to this posture. But, between Middle Ages and Renaissance, new genres use parody, and among them, the drama. Our understanding of parodic plays implies the knowledge of textual but also contextual structures. Pierre de Lesnauderie's plays, performed in Caen in 1493 and 1496, are not only an example of academic performance in France. They both show a complex parodic style, based on the usual ironic pastiches of classical mythology and religious literature, and on satirical imitation of the theatrical structures themselves. The public debate is on stage: an academic riot against the central power; the epidemy of French disease in Europe. Giving new perspectives to parody, the humanistic french drama aims to constitute himself as a political and cultural authority.
\end{abstract}

Résumé: Généralement décrite comme un phénomène textuel, travestissement comique d'ouvrages qui font autorité, la parodie est une écriture particulièrement prisée dans l'Occident médiéval. Cependant, entre Moyen Âge et Renaissance, de nouvelles écritures utilisent la parodie, tel le théâtre dont la double nature de performance et de texte complexifie les usages traditionnels. Pour comprendre la dimension parodique sur scène, il est nécessaire d'enquêter sur un contexte autant que sur des textes. Les pièces de Pierre de Lesnauderie, jouées à Caen en 1493 et 1496, illustrent une pratique universitaire de la scène. Elles font appel à la parodie classique des sources religieuses et littéraires, mais aussi à une satire des événements d'actualité, ce qui conduit à des réflexions novatrices sur les structures théâtrales contemporaines. Sur scène se joue le débat public: une révolte universitaire contre le pouvoir central; la première épidémie de syphilis en Europe. Faire de la parodie une performance comme le propose l'humaniste Lesnauderie, c'est revendiquer - pour le théâtre et pour luimême-, une nouvelle autorité politique et culturelle.

Satire is a lesson, parody is a game. V. Nabokov, Strong Opinions ${ }^{l}$.

Au commencement était le Verbe. La parole de l'Évangile de Jean, I, 1 pourrait servir de prolégomènes, partiellement parodiques, à la définition de la parodie; définition mouvante dont témoignent les recherches critiques du $\mathrm{XX}^{\mathrm{e}}$ siècle, de Bakhtine à Genette. Sa description comme subversion d'une autorité textuelle dans une intention comique ne permet pas d'exprimer la complexité des phénomènes d'imitatio qui s'y conjuguent. Écriture de la déformation construite à

${ }^{1}$ New York, McGrauw Hill, 1973, p. 75.

Cahiers de Recherches Médiévales, 15, 2008 
partir d'auctoritates livresques, la parodie partage avec le pastiche le travail sur la fonction poétique du langage, là où la satire fonctionne sur un mode référentiel. Elle s'en différencie pourtant par l'intention de travestissement comique ou ironique du texte-source. Mais cette divergence d'intention n'est pas toujours aisée à distinguer, en particulier lorsque le temps a éloigné du lecteur moderne le monde culturel où les références prenaient sens. Paroidia, le terme grec entre en français, selon les dictionnaires, au XVII ${ }^{\mathrm{e}}$ siècle ${ }^{2}$. Le geste est pourtant loin d'être moderne comme en témoignent de très nombreux textes médiévaux. Décalage de mots, jeu sur les règles, la parodie ne peut en effet que proliférer dans un univers culturel où la création divine est d'abord invention de signes ${ }^{3}$. Images en marge des manuscrits, chapiteaux des jardins monastiques, centons ironiques de mots ou de notes musicales: l'imposition du centre appelle l'expression des périphéries. Quels renseignements les jeux de l'encodage nous livrent-ils sur les capacités de décodage du public ancien, donc sur des univers culturels aujourd'hui disparus?

Paroidia: en Grèce antique, elle pourrait avoir été un phénomène dramatique, une chanson placée avant ou après l'action, pour la commenter ou la réfuter. Étymologie erronée sans doute, mais qui invite à une exploration. Encore que la question «qu'est-ce que le théâtre au Moyen Âge ?» soit loin d'être résolue, notamment concernant les épineuses relations entre le texte qui nous est parvenu et la performance qui a fait que ce texte a été, peut-être, théâtre, il semble admis aujourd'hui que la scène duplique fréquemment les sources religieuses ou rhétoriques. Or l'étrange objet que nous lisons est une armature de mots privée de performance, de public, de lieux. Si la parodie est un fait textuel, quel est son rôle face au contexte qui définit avant tout le phénomène théâtral ? La connaissance du contexte peut-elle, en retour, nous offrir des indications sur une utilisation spécifique du parodique en scène?

Si le théâtre se diffuse surtout après 1450 dans les pays de langue française, c'est que ce phénomène, aujourd'hui considéré comme médiéval, témoigne d'une civilisation renaissante où la vie publique laïque recherche des expressions de plus en plus nombreuses. La représentation dramatique est souvent bien plus qu'un spectacle ou que le reflet d'une situation concrète; elle appartient à cette situation qu'elle peut même créer. Ce que nous avons choisi de nommer le «théâtre» de Pierre de Lesnauderie est la production de deux pièces à Caen, entre 1493 et 1496. Docteur en droit civil et canon, deux fois recteur de l'Université de Caen en 1505 et 1520, Pierre de Lesnauderie (1450-1522) appartient à ce que l'on appelle communément l'humanisme universitaire. Si la Louange de Mariage et le Recueil des Hystoires des bonnes, vertueuses et illustres femmes est son ouvrage vernaculaire le plus diffusé au début du XVI $\mathrm{XV}^{\mathrm{e}}$ siècle, deux pièces, la Farce de PattesOuaintes $^{4}$ et la Cène des Dieux ${ }^{5}$ ont ouvert, en quelque sorte, sa carrière dans les

\footnotetext{
${ }^{2}$ On le trouve, vers 1614, avec le sens de «imitation bouffonne d'un chant poétique » selon le Trésor de la Langue Française.

${ }^{3}$ L'ouvrage fondateur de P. Lehmann, Die Parodie im Mittelalter (Munich, Drei Masken Verla, 1922) s'ouvrait déjà sur le constat que l'écriture parodique est une tentation permanente à une époque où la cohérence des cadres textuels et mentaux ouvre à la déviance.

${ }^{4}$ La Farce de Pattes-Ouaintes est conservée dans le manuscrit du Matrologe de l'Université de Caen, Caen, Musée des Beaux-Arts, coll. Mancel 69. Le texte, accompagné des documents
} 
lettres. Leur création, en février 1493 pour la première, probablement vers 1496 pour la seconde, indique la présence d'une tradition de représentation par l'Université de Caen et dans le monde scolaire de cette région. Charles de Bourgueville de Bras, ancien étudiant du collège du Bois au début du $\mathrm{XVI}^{\mathrm{e}}$ siècle, en témoigne dans ses Recherches et Antiquités de l'Université de Caen :

De chascun college, la veille des Roys, aucuns sergens et escoliers jouoyent aux carrefours de la ville des farces, dedans des charrettes et sur des chevaux, qui servoient de semonces et invitations pour aller voir jouer, le jour des Roys, des moralites et farces joyeuses auxdits colleges l'apres disner ${ }^{6}$.

Le théâtre est donc une expression ancrée dans un temps festif, indiquant le caractère potentiellement parodique des pièces. Un large public était convié à ces fêtes qui pouvaient avoir lieu, soit dans les locaux des institutions responsables, soit dans des lieux urbains comme le parvis de la cathédrale Saint-Pierre.

Le calendrier des saisons théâtrales n'explique pourtant que partiellement la production de Pierre de Lesnauderie lors du Carnaval de $1493^{7}$. En réponse à une imposition ou decime commandée par le roi de France sur les suppôts de l'Université de Caen, dépouillant ceux-ci du statut financier exceptionnel que leur avait offert au $\mathrm{XV}^{\mathrm{e}}$ siècle le fondateur de l'Université, le roi d'Angleterre, une violente opposition agite les milieux académiques. L'intervention de l'évêque de Châlons en faveur de l'arrêt royal, proposant d'excommunier les contrevenants, est jugée insupportable par l'administration universitaire, d'autant plus que le prélat est un ancien étudiant caennais. Tentant de mobiliser la communauté académique normande, le conservateur de l'université fait placarder, à la cathédrale et «aux lieux eminens de la province de Rouen », des « inhibitions », textes latins et français qui relatent en vers ironiques l'indignité de la politique royale et du traître Châlons.

de l'affaire où il a pris place, est conservé dans les fol. $305 \mathrm{r}^{\circ}$ à $331 \mathrm{r}^{\circ}$. Il a été édité, en 100 exemplaires, à Evreux par Th. Bonnin en 1843. M. Rousse signale également l'existence d'une édition éventuelle de Le Merer, peut-être à Nantes vers la même période, mais il nous a été pour l'instant impossible de la retrouver (M. Rousse, "Gouberville et le théâtre populaire » dans Le Cotentin au temps du sire de Gouberville, Revue du département de la Manche, ${ }^{\circ} 28,1986$, p. 107-118). Nous remercions ici chaleureusement J. Koopmans qui, en nous donnant accès à ces documents, a été à la source de ce travail.

${ }^{5}$ La Cène des Dieux, dans Le Recueil Trepperel, II, les farces, éd. E. Droz \& H. Lewicka, Genève, 1961, p. 97-144.

${ }^{6}$ Les Recherches et Antiquités de l'Université de Caen, Caen, 1588, $2^{\mathrm{e}}$ livre, p. 227. Cette activité est particulièrement décrite dans l'ouvrage ancien d'H. Prentout, La vie de l'étudiant à Caen au XVI siècle, Caen, 1905, qui consacre quelques pages à l'activité de Pierre de Lesnauderie (p. 43-50).

${ }^{7}$ Sur le contexte universitaire et ses relations au théâtre, à Caen et Toulouse, cf. J. Koopmans : «Les universités contre le roi: Caen 1492, Toulouse $1507 »$, dans Das Theater des Mittelalters und den frühen Neuzeit als Ort und Medium sozialer und symbolischer Kommunikation, éd. Ch. Meier, M. Meyer, C. Spanily, Münster, Rhema, 2004, p. 229-236. Il s'agit, à notre connaissance, de la seule étude du contexte de production de la Farce de Pattes-Ouaintes. 
L'évêque de Coutances, lui aussi suppôt de Caen, signifie aux hommes chargés de collecter l'impôt honni leur excommunication. De placards injurieux en procès devant l'Échiquier de Normandie, la querelle se répand dans toute la région, suscitant la gêne du pouvoir central qui ne souhaite pas heurter la sensibilité normande, ni voir se diffuser l'affaire devant le pape. Le lieutenant du bailli, Girard Buriau, mène cependant une action opiniâtre pour collecter l'impôt. Son arrestation maladroite de certains personnages déclenche l'émeute :

Mes comme on devoit aller a la cohue il se trouva a Saint Pierre de Caen bien cinq cent escoliers ou viron aveusques ledit conservateur devant ledit Buriau lequel fut tout troublé et cuidoit estre mort (...) et a telle heure fut prins et rescoux maistre Pierre de Lesnauderie et furent les seigneurs un peu battus devant les grans escolles ${ }^{8}$.

Les jeunes maistres, Lesnauderie, Jean de Caux, deux neveux du conservateur de l'université et d'autres, prennent l'initiative. La tradition dramatique liée aux Jours Gras les pousse à proposer une manifestation polémique sous la forme d'une farce, dont le caractère spectaculaire exprime la menace qu'ils entendent faire peser sur Buriau et ses sbires.

Une farce nommée Pates-Ouaintes, qui fut jouée à Karesme pernant, touchant la decime, et durant la noese d'icelluy. Où il y avoit douze torches et deux faloz ardanz en cordes goctronnées, sans les autres faloz à chandelle. Et y avoit plus de cent escoliers armés et à bastons à la conduire. Et fut jouée devant Buriau qui estoit nommé en icelle Pates-Ouaintes (ibid.).

Il s'agit ici d'un double retournement carnavalesque, les Jours Gras étant normalement l'occasion de satiriser le contexte politique, les acteurs politiques reprenant ici ponctuellement à leur compte la fête pour mêler procession ludique et manifestation armée.

La Farce de Pattes-Ouaintes évoque assez fidèlement le déroulement des événements durant les mois précédents. Mère Université se plaint d'être dépouillée par ses enfants. Quatre suppôts surgissent : Lache-emmanché, dont les protestations de fidélité s'affaiblissent assez vite; Va-t'en-quitte, indifférent à la situation; Escoute-s'il-pleut, qui «nage entre deux eaux » comme il l'indique lui-même (p. 7), et Qui-ne-le-peut-souffrir, seul défenseur fidèle de la Mère. Les personnages incarnent des attitudes différentes parmi les suppôts de l'Université et une recherche historique précise permettrait sans aucun doute de reconnaître, sous le masque de noms à l'apparence proverbiale, des personnalités engagées dans le conflit ${ }^{9}$. Le pasteur dévoyé Ribon-Ribaine, avide de «manger» sa mère comme un loup, est l'image transparente de l'évêque de Châlons. D'abord repoussé, Ribon va quérir Pattes-Ouaintes, qui n'est pas insensible à l'argent. Ce dernier ligote par des édits de papier les mains de l'Université et la livre à Ribon. Qui-ne-le-peut propose d'user

\footnotetext{
${ }^{8}$ Matrologe de l'Université de Caen, fol. 325, $\mathrm{r}^{\circ}$.

${ }^{9}$ Qui-ne-le-peut est ainsi sans doute Ursin Camun, un des universitaires les plus engagés, qui devient vers avril 1493 le nouveau recteur de l'Université. On peut supposer que Lesnauderie offre à son supérieur un hommage que le public savait apprécier.
} 
contre eux du «glaive juridique», c'est-à-dire de l'excommunication. Dans un dernier sursaut, l'Université outragée promet que cet acte provoquera l'indignation « de Dieu puissant, de Pol et Pierre» et qu'il sera vengé par Dieu.

Avant d'être textuelle, la parodie est structurée par un contexte charivarique. Pierre de Lesnauderie joue Pattes-Ouaintes, caricature de Girard Buriau, devant le propre référent de la figure : «Et estoit abilleu comme Buriau, et sy le contrefesoit de parolle et mesmes en sa presence» (ibid.). Les maistres ont-ils joué devant la maison de Buriau? C'est probable. Avaient-ils dressé un échafaud ou simplement délimité l'aire de jeu en l'entourant des porteurs de torches? Il est difficile de le dire. Pattes-Ouaintes finit la pièce en bégayant d'une façon ridicule.

Pattes

Balbutiando sicut Buriau:

Par la mort Dieu metray en prison,

Passe par la ou par la fenestre ;

La chose publique doit estre

Exposée quant besoin en est (p. 25).

À la parole balbutiante s'ajoute l'ironie du message : celui qui annonce sa volonté de «mettre en prison» est prisonnier des universitaires qui lui offrent un miroir grinçant de lui-même, qu'il peut peut-être apercevoir par sa «fenestre». La parodie des paroles du lieutenant dans ce contexte soutient, comme on le voit, un jeu de scène assez mordant.

La Farce de Pattes-Ouaintes est une constante parodie textuelle, composée de citations des pamphlets échangés dans les mois précédents. Un des placards de Saint-Pierre accusait violemment l'évêque de Châlons : In scelere exuperas tragico impia facta Neronis (fol. $304 \mathrm{v}^{\circ}$ ). Dans la pièce, Qui-ne-le-peut déclare à RibonRibaine que «Le fier Néron, monarche et empirant / Tesmoing Seneque, a aymé gens d'estude»( p. 24), signifiant, par comparaison négative, l'indignité de son interlocuteur qui ne respecte pas sa propre mère. Ce procédé de reprise, partiellement masqué par une allusion savante à Sénèque que le texte-source ne proposait pas, permet d'identifier Châlons avec le personnage de Ribon-Ribaine. Or ces pastiches s'effectuent à partir de textes poétiques eux-mêmes largement parodiques. Les auteurs des poésies macaroniques saluaient ironiquement l'évêque du nom de Corydon, emprunté aux pastorales antiques.

O Coridon, Coridon, que te dementra cepit

Que manus herbipotens insigneo carminem facto

Verterit in furia ... (f. 304, $\left.r^{\circ}\right)$

Berger saisi de fureur, mais aussi prêtre oublieux de son devoir, le gardien des agneaux devient loup. La source pastorale caricaturée se mêle au retournement de la célèbre parabole évangélique : le Bon Pasteur égare ici ses brebis avant de les dévorer. Lesnauderie, qui a sans doute été l'un des auteurs des pamphlets, réutilise leur texte parodique comme une métaphore-cadre pour la farce. Il procède d'abord par allusion : dès la page 4, l'Université appelle à l'union de ses suppôts : «Si je 
vous veisse tous ensemble / Je n'eusse pas tel peur des loupz». Puis le thème particularise le personnage félon de Ribon-Ribaine.

Va-t'en-quitte :

Comment, n'est-il pas vray pasteur?

La Mère :

C'est ung gros lou dévorateur

Qui ne lesse rien pendre au clou (p. 8).

Le pastiche du texte-source initial laisse place à un jeu contextuel lui-même parodique : Ribon-Ribaine arbore pour costume une peau de loup.

Qui-ne-le peut :

Mes esse de la peau d'ung lou

Qu'il s'est vestu?

Ribon :

Tout proprement (ibid.).

Selon une mise en scène commune aux théâtres des moralités, des farces et des sotties, l'accessoire incarne la métaphore, en la faisant passer d'une figure à un acte scénique $^{10}$. Caricatures des adversaires, (auto-) citations sur un mode à la fois sérieux et dérisoire, la parodie très diverse pratiquée par Lesnauderie offre à la fois des informations sur la hiérarchie scénique des personnages (jeux de geste, de costume) et permet de percevoir à quel public la pièce s'adresse. L'audience attendue doit connaître dans le détail les divers documents échangés dans les mois précédents, ainsi que le rôle de Lesnauderie ; le public devait donc être universitaire et partisan sans doute les escoliers qui accompagnent cette parade vengeresse.

Avec Pattes-Ouaintes, Pierre de Lesnauderie a choisi d'édifier une redoutable machine de guerre dont la diversité parodique est le moteur. Il sélectionne les images et reprises textuelles mises en scène selon deux critères : des paroles dont la référence autoritaire est complexe (à double source notamment) et dont le rapport satirique au fait contemporain est évident; leur potentiel spectaculaire qui leur permet de devenir jeux de scène. Il y ajoute quelques effets de

${ }^{10}$ Cette utilisation métaphorique des objets scéniques a d'abord été remarquée dans les farces. Cf. M. Rousse, "Les objets facétieux dans les farces françaises des $\mathrm{XV}^{\mathrm{e}}$ et $\mathrm{XVI}^{\mathrm{e}}$ siècles » dans Facétie et littérature facétieuse à l'époque de la Renaissance, $n^{\circ}$ spécial du Bulletin de l'Association d'Études sur l'Humanisme, la Réforme, la Renaissance, 7, 1978, p. 25-29; J. Koopmans et P. Verhuyck, «Les mots et la chose, ou la métaphore comme spectacle. Nouvelle étude sur la représentation scénique de l'acte sexuel dans les farces ", Versants, n³8, 2000, p. 31-51. Pour l'allégorisation polémique et les costumes, nous nous permettons de renvoyer à notre étude: «Finis allegoriae: un trope problématique sur la scène profane française. Nouveaux questionnements sur l'allégorie au théâtre $\left(\mathrm{XV}^{\mathrm{e}}-\mathrm{XVI}^{\mathrm{e}}\right.$ siècles) », Mainte belle oeuvre faicte. Études sur le théâtre médiéval offertes à Graham A. Runnalls, édition D. Hüe, M. Longtin et L. Muir, Orléans, Paradigme, 2005, p. 117-144. 
surenchérissement propres au théâtre, comme le nom des personnages. Ainsi la figure centrale est-elle «la Mère, nommée l'Université ou l'Église» dans le Matrologe. Le scriba conservatoris. Lesnauderie étant l'un des auteurs de ce recueil, cette indécision identitaire semble volontaire. C'est bien une mère éplorée, «veuve et tributaire », " princesse des pays désolée» qui se présente au spectateur dans les premiers vers : l'Université est une autre Rachel, une sœur souffrante de la Vierge, déchirée non par la perte son fils, mais par la trahison des siens. Ce pastiche de l'Ancien et du Nouveau Testament s'ajoute à un autre souvenir textuel: la figuration, habituelle depuis Alain Chartier, de la France soumise à l'invasion anglaise. Cette allégorie de la «dame devenue serve» (p. 4) était largement répandue durant la Guerre de Cent Ans... mais dans le camp français, dont Caen, ancienne capitale des rois d'Angleterre, ne faisait naturellement pas partie. La bataille menée contre la décime royale en 1493 permet aux maistres normands de subvertir la littérature pamphlétaire de leurs anciens adversaires. Enfin la Mère s'adresse au Christ en lui rappelant leurs liens matrimoniaux.

Crist, cher Espoux, lesra tu ton espouze

Estre en ce point par force violée?

Pour quoy veux-tu que aultre que toy m'espouze,

Sinistrement par qui suis defoulée... (p. 25)

Si l'Église est l'Épouse souffrante soumise aux attaques diaboliques, l'Université est aussi fille et épouse du pouvoir royal. Or dans cet appel se glisse la parodie, car Caen se défend d'être, à l'instar de l'Université de Paris, la compagne du roi de France, et appelle à son secours son véritable maître et fondateur, le Christ / roi d'Angleterre ${ }^{11}$.

L'écriture de la Farce de Pattes-Ouaintes par Pierre de Lesnauderie ouvre sans doute à une nouvelle évaluation de ce que pouvait être le phénomène théâtral dans un milieu urbain et universitaire régional. Il serait trop aisé d'opposer carnaval populaire et drame savant des humanistes. La réalité caennaise de 1493 montre que ces distinctions sont largement fausses. Cette pièce renseigne également sur une culture autonomiste très forte chez les intellectuels au début de la Renaissance. Pattes-Ouaintes apporte enfin quelques indices sur la définition problématique de la parodie dramatique. Sur une scène qui prend son sens dans des textes et un contexte précis, les relations entre parodie et pastiche, parodie et satire, parodie et métaphore apparaissent sous le signe du mouvement et de la plasticité de l'action, permettant une forme de polyphonie polémique, entre sérieux et comique.

Probablement lors des Jours Gras de 1496, alors qu'il occupe toujours une position de maistre à Caen, Lesnauderie propose une seconde pièce, d'une envergure et d'une ambition remarquable, la Cène des Dieux. Il s'agit de la première

\footnotetext{
${ }^{11} \mathrm{Au}$ discours biblique dénonçant le viol d'une Mère - Église, Lesnauderie ajoute une référence à la théorie célèbre qui fait, depuis le $\mathrm{XIV}^{\mathrm{e}}$ siècle, de l'Université de Paris l'épouse $\mathrm{du}$ pouvoir royal. Cf. S. Lusignan, Vérité garde le roy. La construction d'une identité universitaire en France, XIII $e^{e} X V^{e}$ siècles, Paris, Presses de la Sorbonne, 1999, p. 135. Le fait que l'Université de Caen ait été créée précisément en 1436 pour contrecarrer l'influence parisienne renforce l'ironie de la référence parodiée.
} 
mise en scène des dieux antiques en France ${ }^{12}$. L'érudition humaniste qui s'y montre semble au premier abord offrir un cadre moins polémique que la Farce de PattesOuaintes. Mais à travers l'imitatio des textes-sources il est possible de distinguer un contexte particulier qui donne sa signification au spectacle.

D'après ses premières éditrices, la Cène des Dieux, issue du Recueil Trepperel, serait un " exemple rare du théâtre de collège ». Lesnauderie proposerait une pièce érudite, mettant en scène des dieux mythologiques, qui sont aussi des planètes. Ce faisant, il rappellerait l'épidémie de peste de 1348 et livrerait le traditionnel message moral sur la vanité du monde et la caducité des êtres. En fait les questions du public, de la représentation, du sens de cette oeuvre demeurent. Est-ce une pièce savante et « humaniste», sans véritable contact avec la vie contemporaine normande? À qui a pu être destinée une mise en scène aussi élaborée ? L'étude des diverses écritures parodiques pourra peut-être nous permettre de cerner plus exactement le spectator in fabula, les intentions de l'auteur et le contexte dans lequel le texte prend son sens.

Saturnus, roi du pays «austral» appelle les dieux à un banquet qui s'avère rapidement un «tribunal» en vue de tirer vengeance des crimes humains. "Une grande cène en son orbe nocturne » (v. 34) se tient donc sous les auspices de Luna : elle réunit Saturne, Vénus, Phébus, Mercure, Mars et Jupiter. La question de la survie de l'homme y est abordée: faut-il le faire mourir ou lui permettre de survivre? Les dieux se constituent en cour de justice. Mercure, le greffier, propose des preuves écrites des vilenies commises depuis la Création. Phébus, Jupiter et Mars se joignent au parti de Saturne. La Vie ne peut convaincre que Vénus. Phébus, annonçant qu'une apocalypse générale demeure entre les mains de Dieu et pourrait affecter le ciel lui-même, conseille d'envoyer aux hommes un "infinable signe» (v. 534), un venin distillé par Junon et qui infectera en quatre ans la société, provoquant le chaos. La Mort se réjouit tandis que Vie se lamente. Junon, prise de pitié, refuse de brasser ce venin. La Mort se rend donc aux Enfers et, aidée d'Atropos, elle recueille l'escume de Cerberus. La Vie, à qui l'on conseille de déserter la terre, décide d'en appeler à la «puissance admirable» de Dieu (v. 1244) contre l'alliance menaçante de Saturnus et Jupiter. La pièce finit par la prophétie que «danger de mort, de famine, de guerre / sont bien prochains, de ce ne ignorent nulz ».

Les 1315 vers de cette pièce, deux fois plus étendue que Pattes-Ouaintes, la placent, selon les estimations de Gratien du Pont, dans l'écriture des «moralités », ce que souligne également son titre dans Trepperel ${ }^{13}$; c'est donc une pièce longue face à l'ordinaire des représentations, particulièrement des farces. Une dizaine de

${ }^{12}$ J. Seznec, dans son ouvrage de référence La survivance des dieux antiques, Paris, 1939, rééd. Flammarion, coll. «Champs », 1993, n'évoque pas ce texte. La seule étude d'envergure qui lui ait été consacrée, à notre connaissance, est l'article de J. Koopmans, «De la survivance des Dieux antiques à la survie de l'humanité ». Nous remercions l'auteur de nous avoir communiqué son travail encore inédit.

${ }^{13}$ « La Cène des Dieux, nouvelle moralité jouée à Caen... ». En 1539, dans L'Art et science de rhetorique metriffiée, Gratien du Pont codifie, à partir de la réalité dramatique des $\mathrm{XV}^{\mathrm{e}}$ et $\mathrm{XVI}^{\mathrm{e}}$ siècles, la longueur des textes scéniques : 200 vers pour un monologue; 500 pour les farces et sotties ; 1000 à 1200 pour les moralités. 
personnages montent en scène, même si seuls environ six ou sept ont une présence scénique commune ${ }^{14}$. Le titre donné à la pièce à l'explicit de Trepperel, Cy finit Cena Deorum alias de convivio Saturni, a attiré l'attention et désigné le drame de Lesnauderie comme un pastiche du Convivio Saturni de Simon de Couvin, rédigé en 1348. Travail intertextuel, dramatisation d'un traité qui pastichait lui-même certains traits de la mythologie antique, la Cène des Dieux semble bien l'œuvre d'un intellectuel humaniste, féru d'imitatio érudite mais qui n'hésite pas à ajouter des scènes «empreintes d'un esprit moqueur et farcesque », selon les éditrices, tirant la pièce vers la parodie.

Il est cependant visible que si la pièce suggère un intertexte assez ancien, elle propose un message portant explicitement sur un avenir proche. Est-ce figure de rhétorique lorsque l'explicit met en garde les spectateurs sur une épidémie en train de se répandre, due à la conjonction néfaste des planètes Saturne et Jupiter ? Tel est, en effet, le déclencheur astrologique de la pandémie en 1348, rappelé par Simon de Couvin. Mais une conjonction du même type a eu lieu vers 1484. Or entre 1495 et 1496 une nouvelle maladie fait son apparition : la syphilis. D'abord très mal connue, elle est sans doute diffusée en Italie par les soldats de Charles VIII ${ }^{15}$. Rapidement l'Europe entière est atteinte et la peur d'une autre grande peste surgit, d'autant plus que la maladie ne trouve que peu de remèdes. Quelques textes littéraires se consacrent à la nouvelle épidémie à partir de $1496^{16}$. La Cène des Dieux n'est donc

${ }^{14}$ Selon M. Rousse, une troupe compte en général six acteurs (Le Théâtre des farces en France au Moyen Âge, tome 2: la représentation, thèse inédite de l'université de Rennes, 1980, p. 98). Pattes-Ouaintes présentait sept personnages. L'incipit du Recueil Trepperel rappelle les noms des principaux acteurs pour la Cène des Dieux : «le général sainct Loys, maistre Jean de Caux, Maistre Pierre de Lesnauderie et leurs compaignons »; il s'agit, pour la plupart, des acteurs mentionnés lors de la représentation de la farce. Le général «Biaunes » et «sainct Loys » sont sans doute des titres portés au sein de compagnies joyeuses, peut-être en rapport avec le monde universitaire.

15 J. Arrizabalaga, J. Henderson \& R. French, The Great Pox. The French Disease in Renaissance Europe, New Haven - Londres, 1997. Les auteurs de cet ouvrage très documenté n'étudient pas, malheureusement, la réception par l'opinion française de cette pandémie et n'évoquent pas la pièce de Lesnauderie, ce qui nous prive pour l'instant de nombreuses informations sur son contexte précis, notamment en Normandie.

${ }^{16}$ Dans le domaine allemand, Sebastian Brandt est le premier à l'évoquer, dans quelques vers dédicacés à Reuchlin et inspirés de la Tragopodagra de Lucain. Joseph Grünpeck (c.14731532), qui vit dans l'entourage de l'empereur Maximilien et qui sera son historiographe à partir de 1497, reprend le thème dans son Tractatus de Pestilentia Scorra sive Mala de Franzos, paru à Augsbourg à la fin de 1496. Il développe une réflexion astrologique et littéraire sur l'épidémie, liant ses causes astrologiques potentielles, la métaphorisation des planètes par les dieux antiques, et un appel moral à la guérison mentale et intellectuelle des Européens. L'édition allemande présente les dieux sur des chariots dont les roues peintes allégorisent la course des étoiles. De tels chariots étaient fréquents dans les manifestations spectaculaires des Habsbourg. L'illustrateur montre-t-il des processions réelles ou s'inspire-til simplement d'une tradition de mise en scène (J. Arrizabalaga et alii, p.111) ? Lesnauderie aurait-il pu avoir un accès au traité de Grünpeck? Il reste difficile de le préciser pour l'instant. 
pas pur plaisir d'érudition; c'est au contraire, à l'instar de Pattes-Ouaintes, une pièce d'actualité, écrite lors d'une crise contemporaine qui touche toute l'Europe. Il semble alors que la pièce date, non de 1492, mais sans doute de 1496 ou 1497, ce qui fait d'elle un des premiers témoignages littéraires connus en France sur ce problème $\mathrm{e}^{17}$.

Au commencement il n'y a donc pas l'intertexte, mais le contexte, comme dans la Farce de Pattes-Ouaintes. Si une lecture attentive du texte ouvre à la découverte de l'univers où le drame est produit, inversement celui-ci éclaire la reprise textuelle comme pastiche ou comme parodie. Le travail sur le texte de Simon de Couvin n'est pas la source de la production de Lesnauderie, mais un outil de commentaire, qui établit un double sens de lecture: les personnages mythologiques sont des planètes, causes premières de la pandémie, et les acteurs du drame contemporain qui se joue dans une Olympe christianisée. La reprise que Lesnauderie fait de Couvin est d'autre part sélective. Seuls deux thèmes sont exploités, le dédoublement des astres en dieux, ce qui lui permet de faire jouer une allégorisation fréquente sur scène, mais ici rendue originale par l'intertexte mythologique; l'interprétation de l'épidémie en termes de punition et la position de prophétie que revêt alors la parole dramatique. L'intertexte est donc utilisé dans la mesure où il offre les éléments les plus propres à construire l'efficacité spectaculaire.

Une reprise textuelle peut cependant en cacher une autre, et le pastiche la parodie. Pour être efficace, Lesnauderie propose une reprise des modèles dramatiques contemporains, beaucoup plus propres à susciter l'attention du public. Le double discours astrologique / mythologique, offert par l'intertexte de Couvin, est amplifié par une double scène banquet / procès, typique des mystères. L'ouverture par Saturnus est un travestissement des exordes infernaux récités par Lucifer ou Satan: en ses "palais obscurs et blesmes» se brasse une nourriture infernale «de vieus serpents qu'ay nourris soirs et mains» (v. 27). Le banquet infernal qui s'ouvre alors est à déchiffrer selon une double grille de lecture empruntée aux scènes contemporaines des mystères. La «cène» est le lieu d'une cuisine maléfique, celle des diables et surtout de tyrans burlesques, comme Tailleboudin et Riffleandouille ${ }^{18}$. Les recettes ridicules et menaçantes qui s'y préparent sont donc objet de rire et d'inquiétude : Saturne appelle ses commensaux à «manger jusqu'à la nappe» et se plaint de la joyeuse compagnie de Vénus et de ses « chants et ris », qui ne cadrent guère avec un décor « empoisonné », « venimeux » et des «brouetz infects» (v. 41, 87, 97-98). Mais les dieux sont aussi les instruments d'une justice divine. Ce repas pris avant la chute parodie évidemment la Cène christique, tout en pastichant sur ce point les repas infernaux des mystères. Ce qui se joue dans cette assemblée n'est pas la rédemption mais la damnation de l'humanité.

\footnotetext{
${ }^{17}$ J. Koopmans propose une datation entre 1494 et 1497, que nous partageons. Proposer 1494 est séduisant car un rythme annuel des productions de Lesnauderie semble alors se dessiner; mais en l'absence de témoignage sur la situation française entre 1494 et 1495, nous serions encline à supposer une représentation vers 1496 ou 1497, alors que le monde intellectuel européen commence à commenter ce sujet.

${ }^{18}$ Cf. J. Koopmans, Le théâtre des exclus au Moyen Âge, hérétiques, sorcières et marginaux Paris, Imago, 1997, p. 164.
} 
Jupiter :

Ennyvrons nous de cest infame

Venin mortel et pestifere,

Affin de tout le monde infere

Sans espargner seigneur ne dame (v. 756-759).

«Le convy triumphant», le partage de la boisson entre des dieux ricanants et gloutons encode une communion inversée où le sacrifice exigé est celui de l'homme. La parodie soutient une ironie amère. Lesnauderie accentue habilement cette impression d'inversion thématique en ajoutant une inversion structurelle : ici la cène, l'un des ultimes actes avant la Passion dans les mystères, ouvre la pièce, amenant au procès devant Dieu, le juge suprême, qui habituellement constitue la scène d'ouverture.

La Cène des Dieux est en effet surtout un procès des hommes. L'inspiration parodique là encore est double, textuelle et structurelle. On sait que, depuis le Mystère de la Passion de Mercadé vers 1435, le procès du Christ devant Pilate se dédouble avec subtilité dans une scène introductive de tribunal au Paradis. Face à un accusateur diabolique, Satan ou Lucifer, qui réclame les âmes des hommes, Dieu juge réunit ses filles, Miséricorde, Justice, Paix et Vérité. Le débat entre les deux premières, alternant des discours de défense et d'accusation, met en question une théologie du salut, à travers un vocabulaire juridique ${ }^{19}$. De ce pastiche qui fait fonctionner efficacement les drames de la Passion, l'auteur propose une parodie : les dieux s'affrontent autour d'un registre où sont notées les actions humaines et qui est, pour son greffier Mercure, «vray teste de Bible» (v. 364). Jupiter est un lâche indécis, déchiré entre les plaintes de Vie, qui s'adresse à sa pitié et à son honneur, et son horreur des actes humains.

Vita :

Vos hommes sont par foy; hommage et rente

Tiennent de vous, soyez leur pytoyable

Jusque a ce que nature soit contente

Gardés que mort ne leur soit dommageable.

Jupiter :

J'ay tel honte de voir leur vie dampnable

Que je renonce a plaider plus leur cause.

Vita :

Ou auray-je recours?

Jupiter :

Allez au diable ! (v. 386-392).

Le dramaturge ajoute également des parodies plus ponctuelles. Au lieu du Christ envoyé sur terre, c'est Mort qui descend de l'Olympe (v. 772). Sa recherche

\footnotetext{
${ }^{19} \mathrm{~J}-\mathrm{P}$. Bordier, Le Jeu de la Passion. Le message chrétien et le théâtre français (XIII ${ }^{e}-X V I^{e}$ siècles), Paris, Champion, 1998, p. 191 et suiv.
} 
du venin de Cerberus semble superposer un enfer antique à l'imaginaire chrétien. Mais autant qu'une référence savante, il y a là une parodie précise des mises en scène de mystères. L'Enfer n'y est-il pas représenté comme une gueule d'animal maléfique, souvent de chien ${ }^{20}$ ? Cerberus, « faulx chien aheurté» (v. 788) donne vie à l'habituel décor infernal. L'inversion ironique de la visite du Christ aux Limbes pour délivrer les sages de l'Antiquité s'ajoute à l'imitation spectaculaire des «secrets» infernaux, effets spéciaux qui se résument ici à un vomissement incontrôlé («issu de la gueulle et des nés » de Cerberus, v. 830). Si les pièces de Lesnauderie sont complexes, c'est en particulier à cause de cette alternance des tonalités, sérieuse et ironique, due aux divers types d'écritures intertextuelles utilisées par le dramaturge.

Le texte parodique offre aussi des éléments d'appréciation sur le statut littéraire auquel l'écrivain aspire. La Farce de Pattes-Ouaintes comme la Cène des Dieux sont des satires du contemporain, face à des troubles politiques locaux ou une situation européenne inquiétante - tant il est vrai que l'événement local autant qu'international peut inspirer une expression théâtrale "médiévale». Pierre de Lesnauderie, à l'instar de nombreux intellectuels de son temps, est un homme de terrain et de science, de scènes et de livres. Sa dramaturgie s'inscrit dans le geste humaniste que P. Eichel-Lojkine nomme "déviance ${ }^{21}$ : le choix d'une position critique face aux problèmes contemporains s'exprime par le détournement ironique des codes culturels, renversés ou rendus ambivalents, et ce décalage est l'occasion d'une réflexion de l'écrivain sur sa position culturelle. Ce n'est sans doute pas un hasard si Pattes-Ouaintes comme La Cène des Dieux se closent sur des tirades prophétiques: l'université est encore en danger; le salut de l'homme n'est pas assuré. Appels à une réflexion réformatrice que le public doit mener sur lui-même, ils proviennent d'une voix qui mêle connaissance du passé, critique moraliste du présent et analyse lucide de l'avenir. Ces traits sont constitutifs d'une nouvelle figure de l'auctoritas depuis le $\mathrm{XIV}^{\mathrm{e}}$ siècle, celle du satiriste mélancolique et ironique $^{22}$. La Cène des Dieux, dont le propos repose sur un jeu entre astrologie et mythologie, insiste à plusieurs reprises sur le crédit limité que l'on doit accorder à l'influence des astres. Les dieux obéissent en dernier recours au Dieu tout-puissant et le libre-arbitre de l'homme est la cause de sa possible rédemption comme de sa chute :

Mercure :

Et s'ilz disent que nous leur faisons tort, Et qu'ilz ayent devant Dieu appellé,

\footnotetext{
${ }^{20}$ Cette représentation célèbre de la «bouche d'enfer » est encore très nette dans les dessins que H. Cailleaux propose à l'occasion de la Passion de Valenciennes en 1547.

${ }^{21}$ P. Eichel-Lojkine, Excentricité et humanisme, Parodie, dérision et détournement des codes à la Renaissance, Genève, Droz, 2002.

${ }^{22}$ La portée problématique des notions de «satire» et de "parodie» au Moyen Âge a été soulignée dans le récent ouvrage Formes de la critique: parodie et satire dans la France et l'Italie médiévales, éd. J-C. Mühlethaler, A. Corbellari et B. Wahlen, Paris, Champion, 2003 (introduction, p. 13-14). Le statut particulier du satiriste narratif, analyste mélancolique, critique et inspiré, de son temps, se dessine à partir notamment de l'exemple du Roman de Fauvel. Cf. J-C. Mühlethaler, Fauvel au pouvoir, la satire au XIV siècle, Paris, Champion, 1994.
} 
Pour fin de cause et pour dernier ressort

Encor n'est pas leur filé desmelé (v. 1276-1279).

Universitaire, Lesnauderie défend sans doute l'astrologia doctrinalis, dont le monde académique est en charge, contre les prophéties de l'astrologia judicialis et la Cène aux dieux ambigus est sans doute un discret témoignage sur ce débat contemporain. Mais les astres en scène servent peut-être un but supplémentaire. Le regard oblique que la parodie porte sur le présent, la tonalité prophétique de l'ensemble sont identiques, chez le dramaturge, aux signes par lesquels le narrateur satiriste revendiquait un èthos exceptionnel, entre engagement et réflexion face au débat public.

Du charivari de rue au tribunal céleste, le parcours qui mène de PattesOuaintes à la Cène des dieux est une exploration de la dimension herméneutique que la scène offre à l'écriture parodique. Le contexte d'une pièce "médiévale» est souvent loin d'être anecdotique. Une grande partie de la production dramatique, entre 1450 à 1550 , est politique, ancrée dans une vie publique, locale, nationale ou internationale, à laquelle elle participe pleinement. Le théâtre que l'on nomme humaniste n'est, alors, pas toujours celui que l'on croit. Est-ce l'imitatio savante de Jodelle à la recherche d'une renaissance du théâtre antique? Est-ce la voix politique de l'universitaire Pierre de Lesnauderie? Quelle que soit l'appréciation littéraire que l'on porte aujourd'hui sur ces diverses productions, il demeure que, là où le poète Jodelle produit du texte en annonçant la hiérarchisation classique entre mots et performance, public populaire et lecteurs savants, le maistre normand propose du théâtre, «médiéval » si l'on veut, où le texte ne fonctionne qu'en constant échange avec un contexte contemporain.

L'utilisation de la parodie indique toujours l'origine d'un discours et l'horizon culturel de ses destinataires. Les pièces analysées dévoilent en France un milieu de production universitaire actif, combatif et se sentant investi d'une mission publique. Elle indique aussi, chez celui qui l'utilise, un rapport particulier à certaines sources littéraires. Elle est souvent un passage vers le discours satirique. Chez Lesnauderie, les textes-sources ne sont pas ridiculisés en eux-mêmes; par leur multiplicité, ils offrent des cadres efficaces à la polémique et font de la scène un espace polyphonique. Mais, parce qu'elle est dramatisée, la parodie s'enrichit de spectaculaire. Or l'efficacité parodique est à son comble lorsque la source de la parodie est une structure dramatique. La scène universitaire s'appuie sur les cadres théâtraux contemporains qu'elle détourne à son profit: temps du carnaval; fonctionnement de la farce; mise en scène des mystères. La parodie au théâtre est une parodie du théâtre. L'auctoritas naît alors de la scène elle-même, prête à se travestir et à utiliser comme horizon de sens une culture dramatique désormais commune à un large public. Dès la fin $d u X^{e}$ siècle, le rire ambivalent des parodies en scène, où le drame s'encode lui-même pour mieux décoder le monde, exprime la maturité d'une écriture.

Estelle Doudet

Université de Lille III 